POS $\quad$ PROCEEDINGS

\title{
COMPASS polarized target in 2018 and 2021
}

\author{
Gerhard Reicherz*, W. Meyer \\ Ruhr-University Bochum, Germany \\ E-mail: gerhard.reicherz@rub.de, meyer@epl.rub.de
}

\author{
N. Doshita, T. Iwata, K. Kondo and G. Nukazuka \\ Yamagata University, Japan \\ E-mail:norihiro.doshita@cern.ch, tiwatalsci.kj.yamagata-u.ac.jp, \\ kaori.kondo@cern.ch, genki.nukazuka@cern.ch
}

\section{Finger, J. Matoušek, and M. Pešek}

Charles University in Prague, Czech Republic

E-mail:miroslav.fingeracern.ch, jan.matousekecern.ch, michael.pesek@cern.ch

\section{J. Koivuniemi}

University of Illinois at Urbana-Champaign, USA

E-mail: jaakko.koivuniemi@cern.ch

\section{Y. Kisselev}

JINR, Dubna, Russia

E-mail: Yury.Kiselev@cern.ch

The COMPASS collaboration performed a polarized Drell-Yan program to measure TMD (Transverse Momentum Dependent) and PDFs (Parton Distribution Functions) in 2015. The program was performed for one more year in 2018 to improve the statistics. In the Drell-Yan program a negative pion beam of $190 \mathrm{GeV} / \mathrm{c}$ with an intensity of $10^{8} / \mathrm{s}$ will be scattered on a transversely polarized proton target with a length of $110 \mathrm{~cm}$. We also plan to use a transversely polarized deuteron target for SIDIS (Semi-Inclusive-Deep-Inelastic-Scattering) program with muon beam in 2021 just after a long shut down of the CERN accelerators. We will present the improved COMPASS PT system for the 2018 run and present the future plan of the deuteron target in 2021.

23rd International Spin Physics Symposium - SPIN2018 -

10-14 September, 2018

Ferrara, Italy

${ }^{*}$ Speaker. 


\section{Introduction}

COMPASS (Common Muon Proton Apparatus for Structure and Spectroscopy) collaboration, which researches measurements on hadron structure and hadron spectroscopy with high intense muon and hadron beams, started in 2012 the second phase of additional measurements to study the polarized Drell-Yan (DY) process to extract the transvers structure of a proton [1]. With eight parton distribution functions (PDFs), the structure of the proton can be described at leading twist. Only three of them survive after integration over the transverse momentum of a parton $k_{T}$. The other five PDFs depending on $k_{T}$ are called TMD PDFs. Sivers function $f_{1}^{\perp}$ is one of the TMD PDFs and describes the correlation between $k_{T}$ and transverse component of the proton's spin [2]. This function can be measured via DY and Semi-Inclusive Deep Inelastic Scattering (SIDIS). According to the universality [3], the sign of $f_{1}^{\perp}$ measured via DY is reversed to the one measured via SIDIS. A confirmation of this prediction is a crucial test for TMD approach in QCD and one of the main purposes of the polarized DY experiment. The COMPASS collaboration performed a

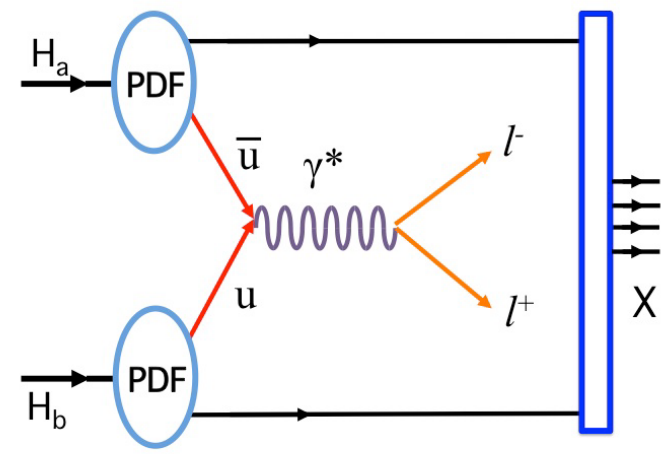

(a) Drell-Yan-Process

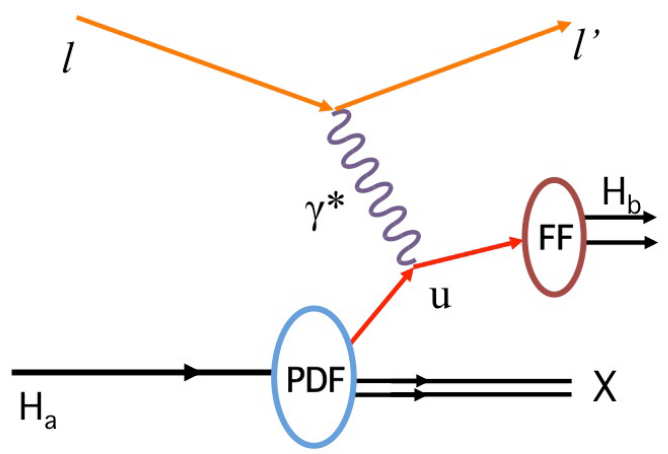

(b) SIDIS-Process

Figure 1: Feynman diagrams of the Drell-Yan and of the SIDIS process.

pilot run in 2014 in order to start in 2015 a full period of 140 days of the measurement of the spin asymmetry of azimuth angle distribution of a dimuon from a pion induced polarized DY process $\pi^{-}+p^{\uparrow} \rightarrow \mu^{+}+\mu^{-}+X$. The $\pi^{-}$with momentum $190 \mathrm{GeV} / \mathrm{c}$ is provided by CERN-SPS. The polarized target provided the transversely polarized proton. The spin asymmetry can be described with a convolution of a nucleon PDF and a pion PDF [6]. The asymmetry related to an azimuth angle $\phi_{S}$, the angle between a transverse momentum of the virtual photon and the target polarization vector, is understood as a convolution of the Sivers function of the proton and a number density function of the pion.

\section{COMPASS polarized target setup}

CERN SPS M2 beam line delivers hadron or naturally polarized positive and negative muon beams in the energy range between 50 and $280 \mathrm{GeV}$. The muon beam polarization is about $80 \%$ at $160 \mathrm{GeV}$. The COMPASS experiment uses those beams with a longitudinally or transversely polarized solid target, liquid hydrogen or heavy nuclear targets 


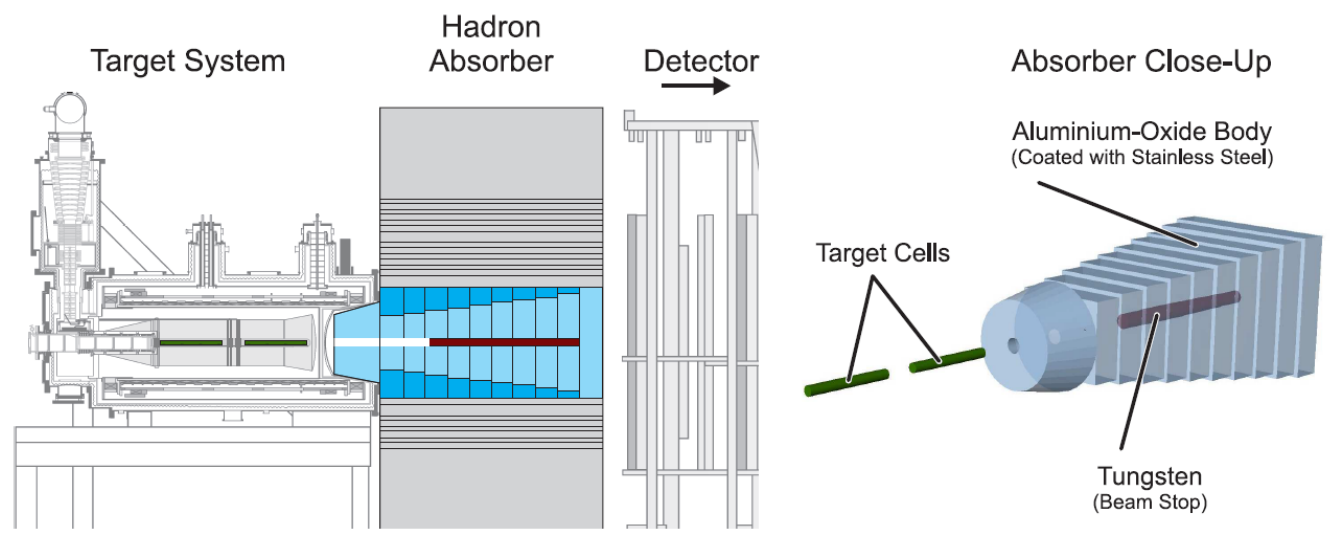

Figure 2: Scheme of the hadron absorber. The left figure shows the position of the absorber in respect to the target system and detector elements. In the right, the main part of the absorber is laid out, with the beam-stop plug and the aluminum-oxide body, together with the respective position of the target cells [13].

The Drell-Yan measurement requires a negative pion beam and a transversely polarized proton target. We use the negative pion beam of $190 \mathrm{GeV}$ mixed with negative kaons and positrons $\left(\pi^{-} 97 \%, K^{-} 2.5 \%\right.$ and $\left.e^{+} 0.5 \%\right)$. Those beam particles are identified by CEDAR (CErenkov Differential counter with Achromatic Ring focus). The CEDAR is placed in the downstream of the M2 line. To increase the vertex resolution the polarized target must be modified. Because of the high hadron beam flux new target cells were constructed. PCTFE was used to provide the high radiation hardness and the avoidance of hydrogen. In 2018 two target cells with $55 \mathrm{~cm}$ in length

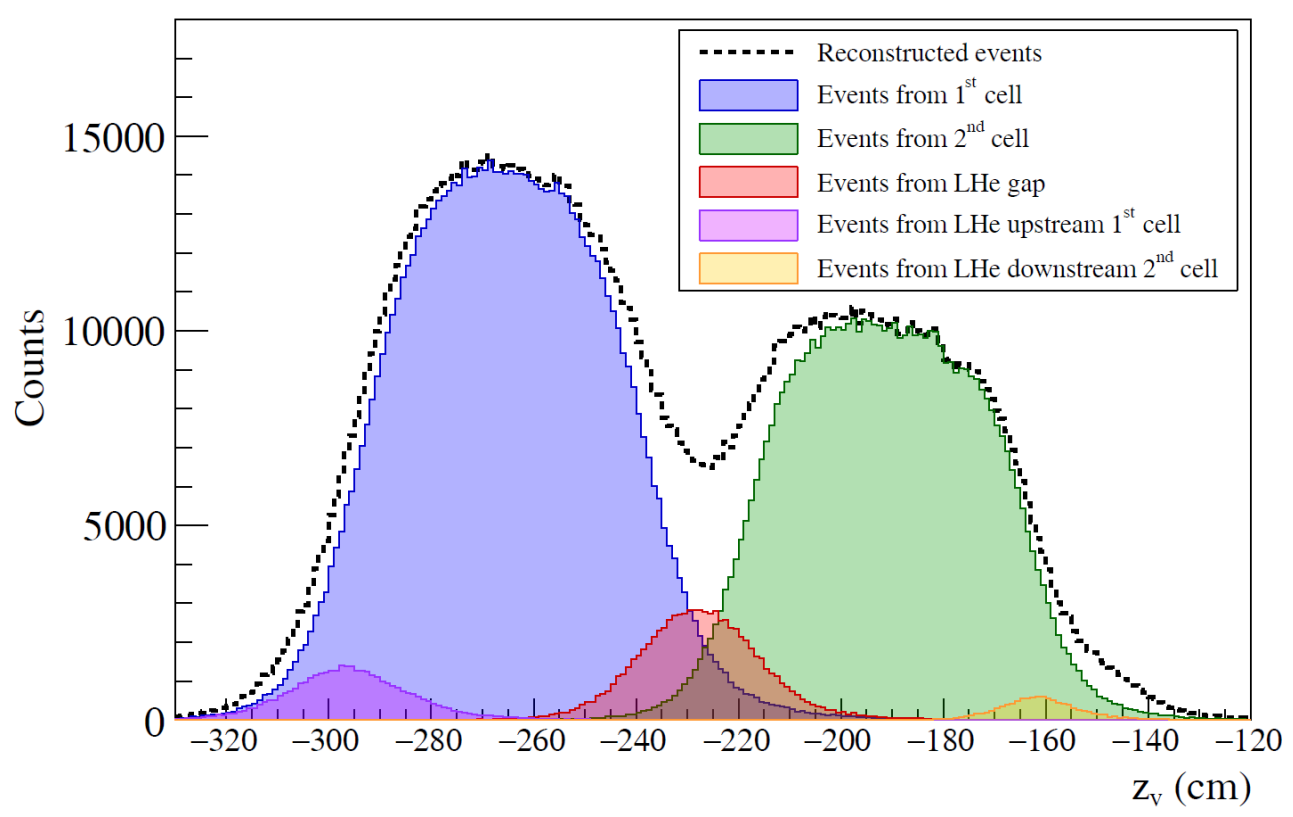

Figure 3: Event migration in the polarized target.

each and diameter of $4 \mathrm{~cm}$ with a cell separation of $20 \mathrm{~cm}$ [7] were mounted. The target was moved $2.3 \mathrm{~m}$ up-stream to install a hadron absorber between target and SM1 (Fig. 2). The installation of 
the hadron absorber will reduce the high secondary particle flux produced by the interaction of the pion beam in the target and, consequently, the tracking detector occupancies. This will make an increase in the intensity of the incident pion beam possible. In figure 3 the event migration in the two cells of the polarized target is shown.

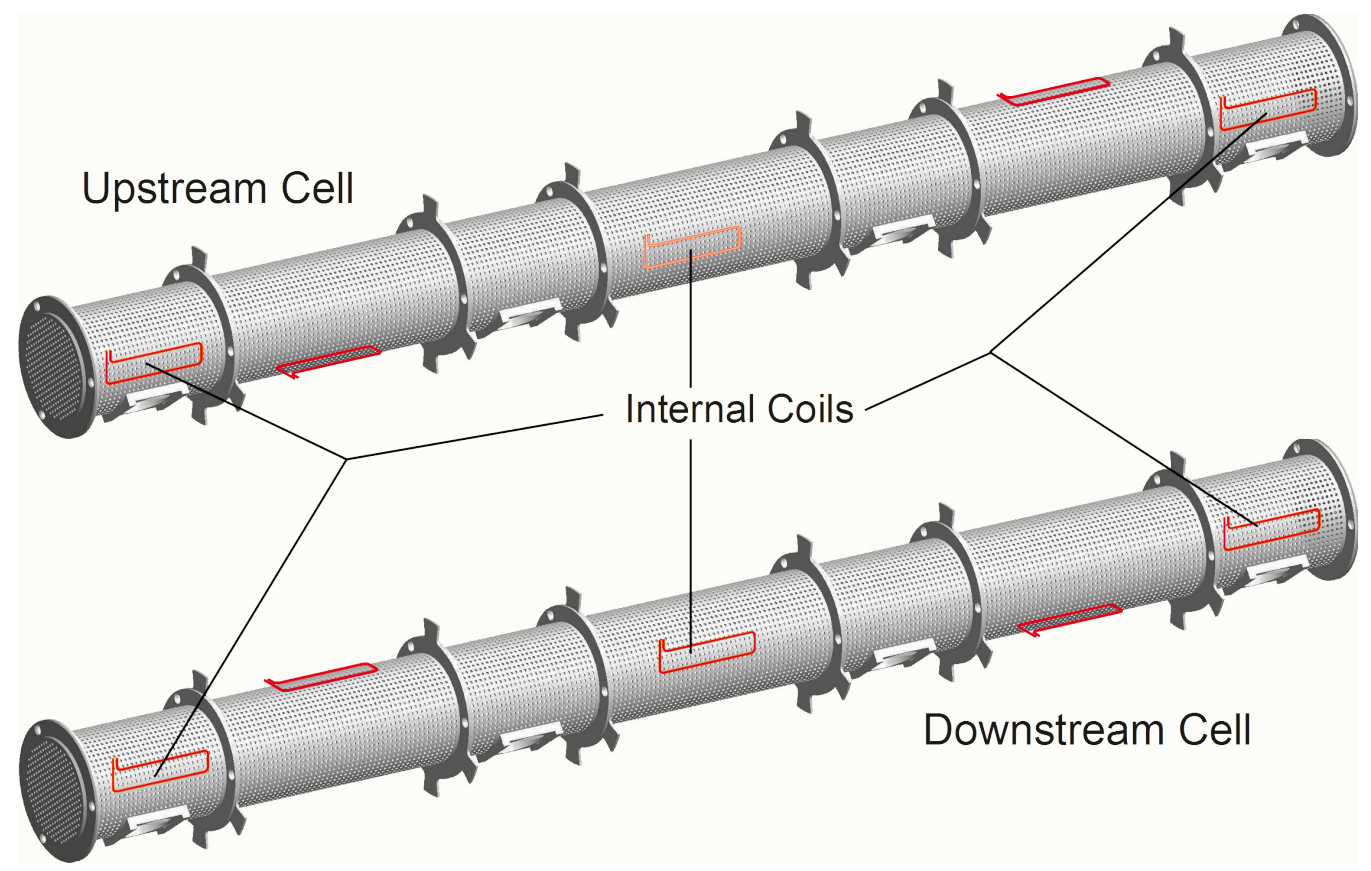

Figure 4: Target cell configuration. Each cell is equipped with five coils, drawn with red line. In 2015 three coils are placed outside and two inside of each cell, respectively.

In 2018 the Drell-Yan measurement will be continued. To measure the target polarization more close to the beam spot, coil 3 and 8 are also mounted into the center of the target cells. Finally 6 coils are mounted in the center of the target cell (beam spot) and four are mounted on the walls. To reduce the cross talk of the neighboring coils, the coils are turned by $90^{\circ}$ from one to the other.

\section{Target materials}

A transverse polarized proton target is needed to study the Drell-Yan process with a $\pi^{-}$beam at $190 \mathrm{GeV}$. Ammonia $\mathrm{NH}_{3}$ is an adequate target material to fulfill the required radiation hardness and has polarization values up to $90 \%$. The ammonia granules were produced in 2011 in Bochum and irradiated with $20 \mathrm{MeV}$ electrons at the LINAC of ELSA in Bonn. In figure 5 the color decay of the ammonia granules are shown at different times after irradiation. The color is not a good evidence for the polarize-ability of the material. The radical concentration reduces to a nearly constant value after 12 month and the maximum polarization values and build-up and relaxation times are very stable, when the material is stored in liquid nitrogen. Recent polarization measurements in Bochum of irradiated ammonia from the production in 1995 and in 2011 are showing a very stable polarization performance. 

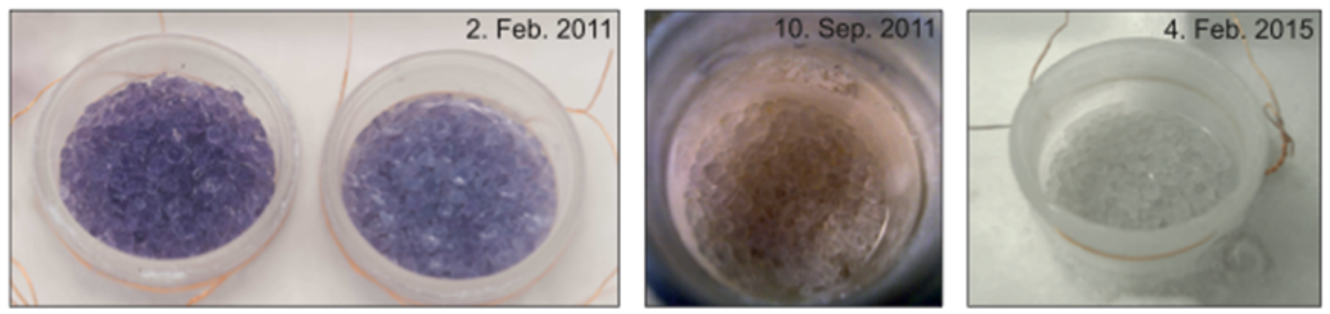

Figure 5: Ammonia granules 1 ween, 2 weeks, 7 months and 4 years after irradiation

To study the SIDIS-process with a muon beam and transverse polarized deuteron target, two materials are preferred deuterated butanol and ${ }^{6} \mathrm{LiD}$. Deuterated ammonia $\mathrm{ND}_{3}$ is also adequate because of the polarization performance but related to the price, production process and irradiation it is not practical to use $900 \mathrm{~cm}^{3}$ of $\mathrm{ND}_{3} .{ }^{6} \mathrm{LiD}$ was already used in phase I of COMPASS in the years 2002 - 2004 and 2006 to study the spin structure of the nucleon. This material can be polarized up to more than $50 \%$ and is stored in liquid nitrogen, what is still an interesting material for future experiments. The Lithium deuterate was produced in Bochum and irradiated at LINAC at ELSA Bonn [4].

In figure $6 \mathrm{~d}$-butanol doped with trityl radical is shown and the polarization versus the $\mu$ wave frequency is plotted. With the use of the trityl radical [14] as dopant in deuterated butanol the polarization was doubled to more than $80 \%$ what results in an increase of the sensitivity for asymmetry measurements by a factor of four. To reach this high polarization values the demands for magnetic field homogeneity is a few times $10^{-5} d B / B$. Butanol is an alcohol what is easy to prepare and to use as target material. The radical concentration is about 2.5 percent by weight and after mixing the liquid is dropped in small beads of about $1-2 \mathrm{~mm}$ into liquid nitrogen. To

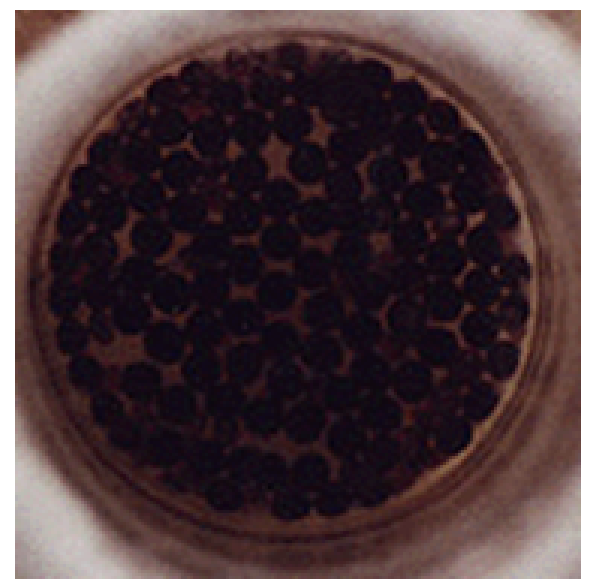

(a) d-butanol doped with trityl

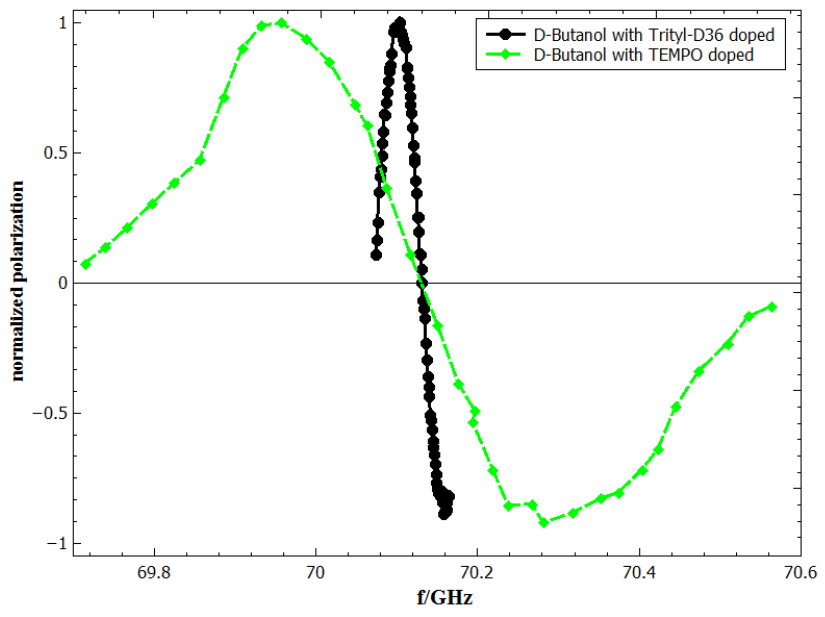

(b) Polarization vs $\mu$-wave frequency

Figure 6: (left) d-butanol doped with trityl radical, (right) polarization vs $\mu$-wave frequency for d-butanol doped with trityl- (black) and TEMPO radical (green)

compare this materials on the accuracy of scattering experiment a view on the Figure of Merit $F$ (FoM) is appropriate. The error in measuring target asymmetry $\Delta A$ depends among other values 


\begin{tabular}{|l|c|c|c|c|}
\hline Material & $P / \%$ & $\rho /(\mathrm{g} / \mathrm{ccm})$ & $\mathrm{f}$ & $F /\left(10^{-2} \mathrm{~g} / \mathrm{ccm}\right)$ \\
\hline d-butanol & 80 & 1.1 & 0.24 & 3.99 \\
\hline$N D_{3}$ & 48 & 1.02 & 0.30 & 2.12 \\
\hline${ }^{6} \mathrm{LiD}$ & 50 & 0.84 & 0.44 & 4.07 \\
\hline
\end{tabular}

Table 1: Figure of Merit for deuterated target materials

on the average polarization $P$, target thickness $\rho$ and the dilution factor $f$, and can be expressed by the FoM $F=f^{2} \cdot P^{2} \cdot n[9]$. The latter factor $f$ is the fraction of polarizable nucleons to the sum of all nucleons in the material. The dilution factor $f=0.44$ for ${ }^{6} \mathrm{LiD}$ can be estimated by a nuclear model picture of ${ }^{6} \mathrm{Li}$ is a three-body $\alpha+p+n$ system [10]. From table 1 can be extracted that FoM $F$ is comparable for d-butanol and ${ }^{6} \mathrm{LiD}$.

\section{Conclusion}

The COMPASS target offers the possibility to polarize proton or deuteron rich target materials up $90 \%$ in longitudinal and transverse direction. The world largest polarized target stands in the power of a few people and in connection with the COMPASS detector system it created and creates an interesting insight to the structure and spectroscopy of the proton.

\section{References}

[1] The COMPASS Collaboration, F. Gautheron et al., COMPASS-II Proposal, CERN-SPSC-2010-014, SPSC-P-340, May 17, 2010.

[2] D. Sivers, Phys. Rev. D 41, 83, 1990.

[3] J.C. Collins, Phys. Lett. B 536, 43, 2002.

[4] J.Ball et al., First results of the large COMPASS ${ }^{6}$ LiD polarized target,Nucl. Instr. Meth. Phys. Res. A 498, 101 (2003)

[5] S. Arnold, A. Metz and M. Schlegel, Phys. Rev. D 79, 034005, 2009.

[6] The COMPASS Collaboration, Ph. Abbon et al, The COMPASS setup for physics with hadron beams, Nucl. Instr. and Meth. A 779, pp. 69 \& 115 (2015).

[7] J. Koivuniemi et al., Large COMPASS polarized solid state target for Drell-Yan physics, XVI International Workshop on Polarized Sources, Targets \& Polarimetry, PoS(PSTP2015)015 .

[8] N. Doshita et al., COMPASS polarized Drell-Yan experiment, XVI International Workshop on Polarized Sources, Targets \& Polarimetry, PoS(PSTP2015)040 .

[9] S. Goertz et al., Polarized target material developments at Bonn and Bochum, XVI International Workshop on Polarized Sources, Targets \& Polarimetry, PoS(PSTP2015)009

[10] A.Meier: Ph.D. Thesis, Ruhr-University Bochum, Germany, 2002

[11] J. Matussek et al., Polarised target for Drell-Yan experiment in COMPASS at CERN, part I, Proceedings of the 22nd International Spin Symposium, 2016.

[12] G. Nukazuka et al., Polarised target for Drell-Yan experiment in COMPASS at CERN, part II, Proceedings of the 22nd International Spin Symposium, 2016. 
[13] A. Berlin Polarized solid ammonia targets for the COMPASS experiment at CERN, PHD thesis,Ruhr-Universität Bochum, Universitätsbibliothek,2016.

[14] St. Goertz et al., Highest polarizations in deuterated compounds, NIM A 526, 43-52, (2004) 